\title{
Preferential Formation of Layered Structure of Ionic Liquid at Ionic Liquid Aqueous Solution / Graphite Electrode Interfaces Observed by Frequency-Modulation Atomic Force Microscopy*
}

\author{
Tomohiro Harada, Yasuyuki Yokota, ${ }^{\dagger}$ Akihito Imanishi, and Ken-ichi Fukui ${ }^{\ddagger}$ \\ Department of Materials Engineering Science, Graduate School of Engineering Science, \\ Osaka University, 1-3 Machikaneyama, Toyonaka, Osaka, 560-8531, Japan \\ (Received 16 December 2013; Accepted 2 February 2014; Published 8 March 2014)
}

\begin{abstract}
Local structure of ionic liquid (IL), 1-butyl-3-methylimidazolium tetrafluoroborate (BMIM-BF 4 ), at IL + water mixtures / highly-oriented pyrolytic graphite (HOPG) interfaces has been investigated using frequency-modulation atomic force microscopy (FM-AFM). The imaging study of surface topography and energy dissipation of IL + water / HOPG interfaces suggested that even the hydrophilic IL molecules favored the HOPG substrate forming stable layers of ILs whose layer thickness corresponded to the averaged ion-pair size. A threshold molar ratio $\left(\chi_{\mathrm{IL}}\right)$ for the first IL layer formation was between 0.01 and 0.05 and the HOPG surface was completely covered by IL layer at as low as $\chi_{\mathrm{IL}}=0.1$. Force curve measurements in various concentrations of IL + water mixtures also suggested that the liquid side nature such as the apparent viscosity near the interfaces changed around the critical concentration. Dissolution of the IL layer was found to be a slow process, suggesting that the interface structure was history-dependent. Furthermore, electrochemical potential of the HOPG substrate affected the IL layer formation. Particularly application of positive potential resulted in growth of IL layer at the interface.
\end{abstract}

[DOI: $10.1380 /$ ejssnt.2014.89]

Keywords: Atomic force microscopy; Electrochemical methods; Energy dissipation; Graphite; Solid-liquid interfaces; Ionic liquids

\section{INTRODUCTION}

Ionic liquids (ILs) have attracted much attention as designable liquids because of their unique structures and properties like low vapor pressure, non-flammability, thermal stability, and wide electrochemical window [1]. Especially the information of ILs / solid interfaces is much important for various applications such as electrochemistry [1, 2], fuel cell [2], dye-sensitized solar cell [3], organic field effect transistors [4], lubrication [5], and heterogeneous catalysis [5]. The detailed knowledge about ILs / solid interfaces improves the efficiency and selectivity for their applications. Hence many groups have investigated these interfaces using various methods, for example, sum-frequency generation [6], X-ray reflectivity [7], atomic force microscopy (AFM) [8-18], scanning tunneling microscopy [9], resonance shear measurements [19], and molecular dynamics simulation [20-25]. These results suggested that the structuring of IL molecules at ILs / solid interfaces was so important, and an in-depth understanding of them is required.

Recently, our group directly observed quite stable layered structure at ILs / highly-oriented pyrolytic graphite (HOPG) and ILs / mica interfaces using frequencymodulation atomic force microscopy (FM-AFM) [26], which can detect and sustain piconewton force. Since the first demonstration of atomic-resolution imaging of mica substrate in water in 2005 [27], FM-AFM in various solutions has become a powerful tool for elucidating not only

\footnotetext{
*This paper was presented at the 12 th International Conference on Atomically Controlled Surfaces, Interfaces and Nanostructures (ACSIN-12) in conjunction with the 21st International Colloquium on Scanning Probe Microscopy (ICSPM21), Tsukuba International Congress Center, Tsukuba, Japan, November 4-8, 2013.

†Corresponding author: yyokota@chem.es.osaka-u.ac.jp

$\ddagger$ Corresponding author: kfukui@chem.es.osaka-u.ac.jp
}

the surface topography of soft materials but also a solvation layer at the interfaces [28-32]. Our group applied this fascinating tool to ILs / solid interfaces in the neat ILs, and revealed that step-terrace structures of IL molecules were formed at the interfaces with negligible IL mobility. In our previous study, we suggested that the step heights of the layered structure corresponded to the ion pair diameter of the IL molecule calculated from cubic root of molecular volume [26].

High viscosity of ILs is known to hamper the application including material transport, and mixing with other solvents is one of the effective methods to decrease the viscosity. Hence the mixtures of IL and water or organic solvents are extensively investigated [33-39]. Jeon et al. showed that the structure of BMIM-BF 4 (1-butyl-3methylimidazolium tetrafluoroborate) in IL + water mixtures changed at the specific water concentration $c_{\text {water }}=$ 32 and $45 \mathrm{~mol} \mathrm{l}^{-1}$ by using attenuated total reflection infrared (ATR-IR) absorption [39]. However, there are not so many experimental reports concerning IL + water / solid interfaces $[40,41]$. Recently, Smith et al. revealed that the profiles of solvation force at the IL + water of EAN (ethylammonium nitrate) / silica interfaces depended on water concentration by force curve measurements based on contact-mode AFM [41].

Although many investigations concerning pure ILs / solid interfaces were reported previously, the information about IL + water / solid interfaces is much important to elucidate the origin of the layered structure of IL molecules at ILs / solid interfaces. In this study, we investigated IL + water / HOPG interfaces using FM-AFM, where IL + water mixtures were composed of BMIM-BF 4 due to its high miscibility to water [42]. Simultaneous measurements of surface topography and energy dissipation revealed that the layered structure of IL molecules at the interface was formed even at quite low concentrations of IL. Furthermore, combining the imaging and force curve studies at various concentrations of IL + water mix- 

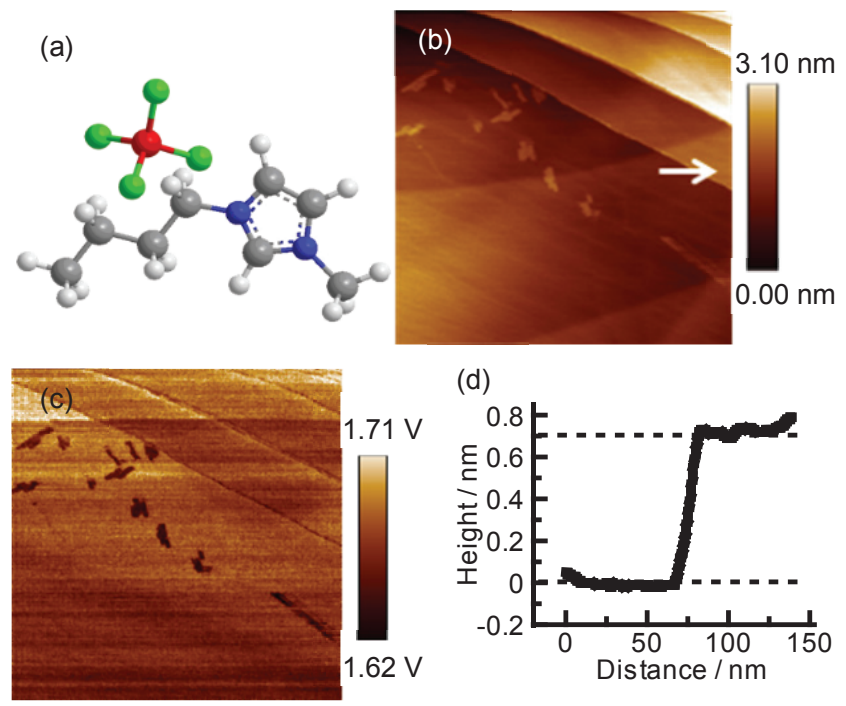

(d)

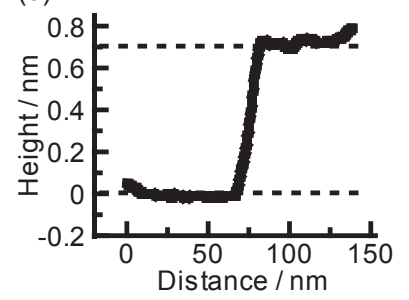

FIG. 1: (a) Molecular structure of BMIM-BF 4 . Nitrogen, carbon, boron, fluorine, hydrogen atoms are indicated as blue, gray, red, green and white spheres, respectively. Typical FMAFM topography image (b) and simultaneously obtained energy dissipation image (c) of an HOPG surface in BMIM-BF4. $1 \times 1 \mu \mathrm{m}^{2}, \Delta f=+75 \mathrm{~Hz}, A_{\mathrm{p}-\mathrm{p}}=0.83 \mathrm{~nm}$. (d) Line profile along an arrow in panel (b).

tures, we propose a schematic representation of the IL + water / HOPG interfaces.

\section{EXPERIMENTAL}

An IL, BMIM-BF 4 (Fig. 1(a)), was purchased from Tokyo Chemical Industry (purity $>98 \%$ ), and used without further purification. Milli-Q water (Nihon Millipore) was used for the preparation of IL + water mixtures.

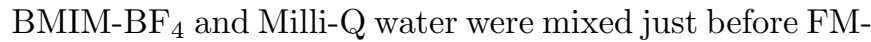
AFM measurements. The HOPG substrate with large grain size was kindly supplied from Prof. T. Enoki and Dr. K. Takai in Tokyo Institute of Technology. Air ambient FM-AFM measurements of as-cleaved surfaces of the HOPG revealed that a step density (average step length per a unit area) was as small as $0.97 \mu \mathrm{m} \mu \mathrm{m}^{-2}$.

FM-AFM measurements were performed using an FMAFM (JSPM-5200 specialized for electrochemical environment, JEOL). To oscillate the cantilever always at its resonant frequency while keeping constant amplitude and to accurately detect the frequency shift $(\Delta f)$ due to interactions between the tip and the sample, the phase locked loop circuit was used. All the FM-AFM images were obtained with a constant frequency shift mode: the AFM tip traces the surface or interface with keeping the constant $\Delta f$. The vibrating cantilever loses its energy even in the homogeneous surroundings but additional lost occurs when dissipative interaction works during the measurements. The voltage which applies to the piezo actuator to oscillate the cantilever at the constant amplitude (peak-to-peak drive amplitude; $V_{\mathrm{Dp}-\mathrm{p}}$ ) is recorded simultaneously with the topography height and visualized as an energy dissipation image. In the present study, the set
TABLE I: Molar fraction $\chi$ and molar concentration $c$ $\left(\mathrm{mol} \mathrm{l}^{-1}\right)$ for each component, calculated density $\rho_{\text {liq }}\left(\mathrm{g} \mathrm{cm}^{-3}\right)$, calculated viscosity $\eta_{\text {liq }}(\mathrm{mPa} s)$ and calculated resonant fre-

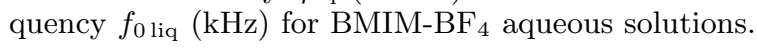

\begin{tabular}{ccccccc}
\hline \hline$\chi_{\mathrm{IL}}$ & $\chi_{\text {water }}$ & $c_{\mathrm{IL}}$ & $c_{\text {water }}$ & $\rho_{\text {liq }}$ & $\eta_{\text {liq }}$ & $f_{0 \text { liq }}$ \\
\hline 0 & 1 & 0 & 55.3 & $0.997^{*}$ & $0.890^{*}$ & 148 \\
0.005 & 0.995 & 0.264 & 52.6 & 1.00 & 0.911 & 148 \\
0.01 & 0.99 & 0.505 & 50.0 & 1.01 & 0.933 & 147 \\
0.02 & 0.98 & 0.931 & 45.6 & 1.03 & 0.978 & 146 \\
0.05 & 0.95 & 1.88 & 35.7 & 1.06 & 1.13 & 144 \\
0.1 & 0.9 & 2.85 & 25.6 & 1.10 & 1.42 & 141 \\
0.2 & 0.8 & 3.83 & 15.3 & 1.14 & 2.28 & 137 \\
0.3 & 0.7 & 4.34 & 10.1 & 1.16 & 3.66 & 133 \\
0.4 & 0.6 & 4.64 & 6.96 & 1.17 & 5.86 & 129 \\
0.5 & 0.5 & 4.84 & 4.84 & 1.18 & 9.39 & 124 \\
0.6 & 0.4 & 4.99 & 3.32 & 1.18 & 15.0 & 119 \\
0.7 & 0.3 & 5.10 & 2.18 & 1.19 & 24.1 & 113 \\
0.8 & 0.2 & 5.18 & 1.29 & 1.19 & 38.6 & 107 \\
0.9 & 0.1 & 5.25 & 0.58 & 1.19 & 61.9 & 99.5 \\
1 & 0 & 5.31 & 0 & $1.201^{*}$ & $99.2^{*}$ & 91.7 \\
\hline \hline Da & & & & & &
\end{tabular}

* Data from Ref. [42].

point value of the frequency shift was positive $(\Delta f>0)$ in liquid and negative $(\Delta f<0)$ in air. Typical deflection noise density and quality factor ( $\mathrm{Q}$ factor) of the cantilever in IL + water mixtures were $25-60 \mathrm{fm} \mathrm{Hz}^{-1 / 2}$ and 1-9, respectively. The peak-to-peak amplitude of the vibrating cantilever $A_{\mathrm{p}-\mathrm{p}}$ and scan speed were set in the range of $0.6-2.3 \mathrm{~nm}$ and $0.477-2.93 \mu \mathrm{m} \mathrm{s}^{-1}$, respectively. The $z$-scale of FM-AFM images was calibrated using the height of the $\mathrm{Au}(111)$ step. Electrochemical FM-AFM measurements were performed under the potential control by using bipotentiostat (AFCBP1, Pine). Pt wires were used as the quasi-reference and counter electrodes.

Conductive $\mathrm{Si}$ cantilevers back-coated by $\mathrm{Au}$ (PPPNCHAuD, NanoSensors, and 300GD, BudgetSensors) with force constants around $40 \mathrm{~N} \mathrm{~m}^{-1}$ were used for the force sensors. The cantilever surfaces were cleaned prior to use by a method proposed by Fujihira et al. [43]. The cantilevers were irradiated by UV light for 20 minutes followed by immersion in hot ethanol $\left(70^{\circ} \mathrm{C}\right)$ for 20 minutes. HOPG substrates were cleaved by an adhesive tape just before dropping the IL + water mixture on them for FMAFM measurements. All measurements were conducted at room temperature.

\section{RESULTS AND DISCUSSION}

Figure 1(b) shows a typical topographic FM-AFM image of an HOPG surface taken in pure BMIM-BF ${ }_{4}\left(\chi_{\mathrm{IL}}\right.$ $=1$ ). Although BMIM-BF ${ }_{4}$ has high viscosity (Table I), FM-AFM measurements were possible and the presence of a layered structure of IL molecules with rather straight steps was observed as reported previously [26]. The step height (one layer thickness) of $0.68 \pm 0.12 \mathrm{~nm}$ showed good correspondence to the ion pair diameter of $0.69 \mathrm{~nm}$ (the cubic root of the molecular pair volume of the IL [40]) as can be confirmed in a line profile along a step shown in Fig. 1(d). Other than the $0.68 \mathrm{~nm}$ high steps, steps of $0.36 \pm 0.12 \mathrm{~nm}$ high were observed. The height difference of the latter corresponded to the layer separation 


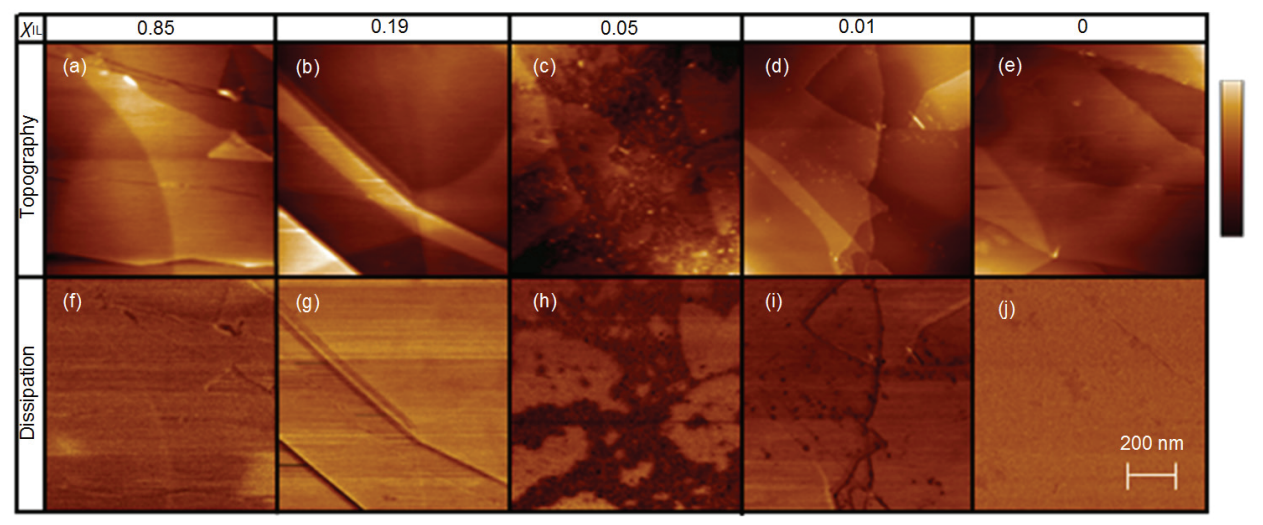

FIG. 2: Topography (top) and energy dissipation (bottom) FM-AFM images $\left(1 \times 1 \mu \mathrm{m}^{2}\right)$ of HOPG surfaces in IL + water mixtures. Measurement conditions: (a), (f) $\chi_{\mathrm{IL}}=0.85, \Delta f=+121 \mathrm{~Hz}$. (b), (g) $\chi_{\mathrm{IL}}=0.19, \Delta f=+152 \mathrm{~Hz}$. (c), (h) $\chi_{\mathrm{IL}}=0.05, \Delta f=+199 \mathrm{~Hz} .(\mathrm{d}),(\mathrm{i}) \chi_{\mathrm{IL}}=0.01, \Delta f=+95 \mathrm{~Hz}$. (e), (j) $\chi_{\mathrm{IL}}=0, \Delta f=+85 \mathrm{~Hz}$. The range of the contrast scale bar for each topography image is (a) $2.70 \mathrm{~nm}$, (b) $2.85 \mathrm{~nm}$, (c) $2.03 \mathrm{~nm}$, (d) $3.30 \mathrm{~nm}$, and (e) $2.75 \mathrm{~nm}$, respectively. The contrast scale bar for each energy dissipation $\left(V_{\mathrm{Dp}-\mathrm{p}}\right)$ image corresponds to (f) 0.97 to $1.01 \mathrm{~V}$, (g) 0.71 to $0.78 \mathrm{~V}$, (h) 0.93 to $1.03 \mathrm{~V}$, (i) 1.46 to $2.70 \mathrm{~V}$, (j) 0.139 to $0.141 \mathrm{~V}$, respectively.

of graphite $(0.335 \mathrm{~nm})$ and probably the IL overlayers on different graphite layers provided such a step height. The structure was quite stable and its morphology remained unchanged during repeatedly scanning the area by the AFM tip. It indicated that the layers neither grew nor shrank at the steps in the presence of many IL molecules in the liquid phase, and the number of the IL layers was not homogeneous on a rather flat HOPG surface [26].

The simultaneously recorded energy dissipation image is shown in Fig. 1(c). One may notice that almost constant dissipation was recorded in the flat terrace regions and no appreciable contrast was observed between terraces across the steps both of $0.68 \mathrm{~nm}$ high and $0.36 \mathrm{~nm}$ high. It suggests the similarity of the composition and the local structure of the flat regions. In contrast, bright protrusions in Fig. 1(b), which were probably agglomerates of IL molecules, gave smaller dissipation (dark depressions in Fig. 1(c)).

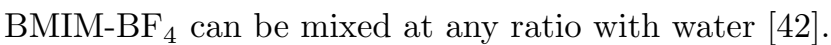
The calculated properties of IL + water mixtures at various concentrations are shown in Table I. See Appendices for details of calculation methods of the density, viscosity of the solutions and the resonant frequency of the cantilever in the solutions, and their comprehensive table (Table S1). Evaporation or condensation of water during FM-AFM measurements are critical issues for imaging in $\mathrm{IL}+$ water mixtures. It is because the FM-AFM method uses the resonant frequency of the cantilever as the feedback signal for the tip height control and the reference resonant frequency without any force from the interface varies depending on the IL molar fraction (Table I). As shown in Fig. S1, the peak frequency of Brownian motion of the cantilever in IL + water mixtures showed good agreements with the expected values. Therefore, the molar fraction was calibrated by the peak frequency of Brownian motion of the cantilever measured just before approaching the tip to the interface for FM-AFM imaging or just after the imaging (See Appendices for details).

Figure 2 shows typical FM-AFM images in some selected concentrations of IL + water mixtures. The top panels are topography images and the bottom panels are energy dissipation images. As far as $0.1<\chi_{\mathrm{IL}}<1$ (Figs. 2(a) and (b)), the observed structure was similar to that for pure IL $\left(\chi_{\mathrm{IL}}=1\right.$, Fig. $\left.1(\mathrm{~b})\right)$; stable solid-like stepterrace structures of IL molecules were formed at the IL + water / HOPG interfaces. The step heights were almost the same as the case of $\chi_{\mathrm{IL}}=1$, namely corresponded to the ion pair diameter calculated by cubic root of molecular pair volume, or the substrate step, as noted above. But the step heights had apparently larger distribution, showing more inhomogeneous layer thickness, as shown in Fig. S2. One of the possible reasons is that the cationic ion takes various conformations because of the presence of water molecules [36]. Moreover, the large steps ( $\sim$ a few $\mathrm{nm})$ were rarely observed as $\chi_{\mathrm{IL}}$ decreased, in contrast to the case of pure IL.

As shown in Figs. 2(f) and (g), there was no apparent contrast across the steps, showing the amounts of energy dissipation were almost the same between the different terraces. Hence each terrace had the same surface nature, that is, the whole surface was covered with IL layers.

In the range of $0 \leq \chi_{\mathrm{IL}}<0.05$ (Figs. $2(\mathrm{~d})$ and $(\mathrm{e})$ ), each topography image also showed a step-terrace structure, whose step heights were mostly $0.35 \mathrm{~nm}$, in accordance with the value for bare HOPG. Although some contrast was observed at the step edges, the amounts of energy dissipation were almost the same between the different terraces in Fig. 2(i) $\left(\chi_{\mathrm{IL}}=0.01\right)$. IL agglomerates were observed as depressions in Fig. 2(i), as is the case in Fig. 1(c). Thus a bare HOPG surface with some IL agglomerates was observed for $\chi_{\mathrm{IL}}<0.05$. It is noted that imaging of the interface was quite difficult for the range of $0.005<\chi_{\mathrm{IL}} \leq 0.01$ in spite of many trials.

At $\chi_{\mathrm{IL}}=0.05$, energy dissipation at two terraces separated by the $0.68 \mathrm{~nm}$-high step was clearly different $\left(\Delta V_{\mathrm{Dp}-\mathrm{p}} \approx 40 \mathrm{mV}\right.$ in Fig. $\left.2(\mathrm{~h})\right)$. It indicated the different surface natures, and we attributed the lower dissipation plane to HOPG substrate (lower terrace in topography) and the higher dissipation plane to the first IL layer (higher terrace in topography). The molar ratio of 

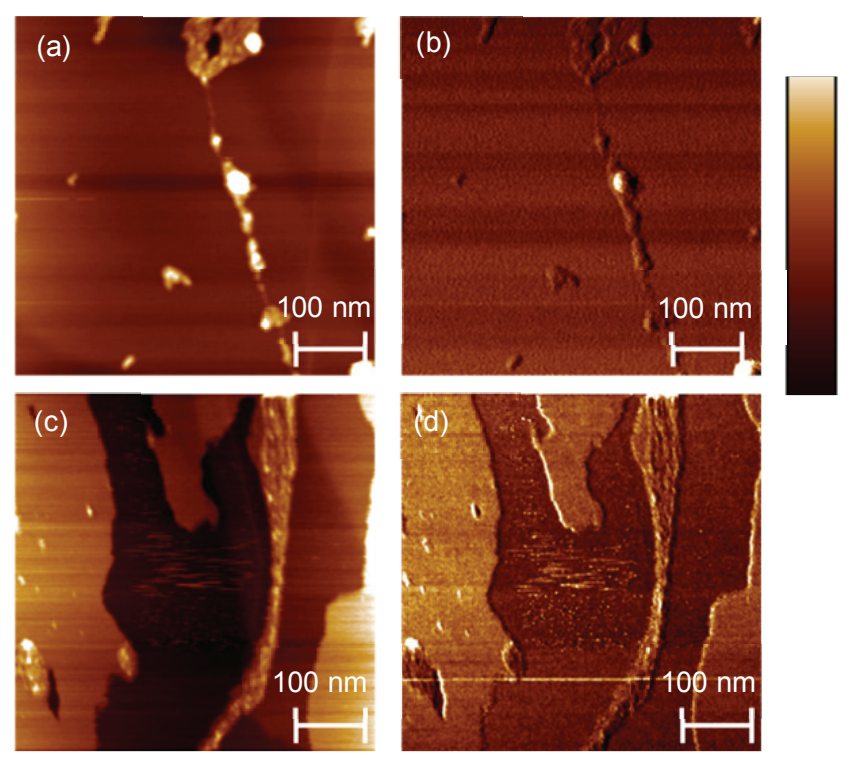

FIG. 3: Topography ((a), (c)) and energy dissipation ((b), (d)) FM-AFM images $\left(500 \times 500 \mathrm{~nm}^{2}\right)$ of an HOPG surface in air after removing liquid phase of IL + water mixtures at two molar fractions. The IL + water mixtures $\left(\chi_{\mathrm{IL}}=0.01,0.05\right)$ was dropped on a freshly cleaved HOPG surface and held it up under saturated water vapor for 10 minutes. Then, the liquid phase on the HOPG was removed by tilting the sample and the surface was imaged by FM-AFM in air. (a), (b) $\chi_{\mathrm{IL}}=0.01$, $\Delta f=-20 \mathrm{~Hz}$. (c), (d) $\chi_{\mathrm{IL}}=0.05, \Delta f=-60 \mathrm{~Hz}$. The range of the contrast scale bar for each topography is (a) $4.37 \mathrm{~nm}$ and (c) $2.36 \mathrm{~nm}$, respectively. The contrast scale bar for each energy dissipation $\left(V_{\mathrm{Dp}-\mathrm{p}}\right)$ image corresponds to (b) 1.78 to $2.04 \mathrm{mV}$, (d) 6.00 to $6.23 \mathrm{mV}$, respectively.

$\chi_{\mathrm{IL}}=0.05$ was close to the threshold of the IL layer formation because the mixture of two surfaces was observed only at this concentration. Another example of the FMAFM image set at this molar ratio is provided in Fig. S3 in Appendices. Contrast difference in the dissipation image was found at a $0.68 \mathrm{~nm}$-high step, but not found at a $0.35 \mathrm{~nm}$-high step.

These interface structures can be exposed to air by removing liquid phase of IL + water mixtures. After removing the $\mathrm{IL}+$ water mixture of $\chi_{\mathrm{IL}}=0.05$ (Figs. 3(c) and $(\mathrm{d})$ ), contrast difference in the dissipation image was found at $0.68 \mathrm{~nm}$-high steps $\left(\Delta V_{\mathrm{Dp}-\mathrm{p}} \approx 0.065 \mathrm{mV}\right)$, but not found at $0.35 \mathrm{~nm}$-high steps (Fig. $3(\mathrm{~d})$ ). Thus, the same results were qualitatively obtained even after removing the liquid phase: energy dissipation is higher on the first IL layer (higher terrace in topography) than on the HOPG substrate (lower terrace in topography). The dissipation value itself was much smaller due to the absence of the liquid phase which costs much energy for the vibration. In the case of the IL + water mixture of $\chi_{\mathrm{IL}}=0.01$ (Figs. 3(a) and (b)), energy dissipation magnitude was constant at flat area of different terraces separated by steps. These steps were $0.35 \mathrm{~nm}$-high steps of the HOPG substrate. It is noteworthy that the IL agglomerates were rather observed as protrusions in energy dissipation images in air (Figs. 3(b) and (d)) in contrast to depressions in the images in IL and IL + water mixtures (Figs. 1 and 2 ). It may reflect site-specific solvation structure of liquid phase molecules at the interfaces.

Several origins of energy dissipation in FM-AFM have been proposed in literature $[28,44]$. For measurements under ultrahigh vacuum (UHV), the possible origins of energy dissipation are considered to be adhesion hysteresis, Joule heating, and the random forces originating from the vibrations of surface atoms [44]. The origins of energy dissipation in liquid measurements are complicated and not fully understood [28]. The local dissipation difference is strongly dependent on the sample nature itself and a solvation structure of liquid phase molecules around the sample and the AFM tip, which is further perturbed by vibration of the cantilever in close proximity of the sample surface.

Dissolution of the IL layer against water was examined to check the time scale of the equilibrium for the interface structure. Starting from the surface that was partially covered with an IL layer prepared from the IL + water mixture of $\chi_{\mathrm{IL}}=0.05$, the liquid phase was replaced with water and the change of the surface structure was measured (Fig. S4 in Appendices). Even after coexistence of much more volume of water compared with the threshold concentration for the formation of the IL layer $\left(\chi_{\mathrm{IL}}=0.05\right)$ for a few tens of minutes, considerable amount of IL remained on HOPG. This result indicates that the reverse process (dissolution of the IL layer) is much slower than the formation of IL layer at the interface.

It is an important issue for the electrochemical applications of ILs how the interface structure changes according to the electrode potential. Figure 4 shows a result of the potential dependence of the interface where the HOPG electrode was partially covered with the single IL layer in the solution of $\chi_{\mathrm{IL}}=0.05$. The electrode potential changed in the range from $-700 \mathrm{mV}$ to $+750 \mathrm{mV}$, where no apparent chemical process occurred judging from cyclic voltammograms of this surface shown in Fig. S5 in Appendices. Starting from the rest potential $(-130 \mathrm{mV})$, switching to $+750 \mathrm{mV}$ resulted in a drastic structural change (Figs. 4(b) and (d)). Four bright protrusions of ca. $3 \mathrm{~nm}$ high appeared and stable imaging became difficult. After the potential was returned to the original value of $-130 \mathrm{mV}$, it was found that most of the surface was covered with a single flat IL layer and unstructured agglomerates of less than $0.9 \mathrm{~nm}$ high on that. Bare HOPG region with lower height in topography and smaller dissipation (darker in image) disappeared. Dark spots at the bottom band of Fig. 4(d) correspond to the protrusions in Fig. 4(b). They are probably due to unstructured IL agglomerates on the IL layer, which were also observed in Figs. 2(c) and (d) and Fig. S3(a) and (b) in Appendices. Extension of the flat IL layer of the ion-pair-size thickness indicates that ion pairs were incorporated on the electrode rather than a preferential adsorption of anions by application of the positive potential of $+750 \mathrm{mV}$. When a negative potential of $-700 \mathrm{mV}$ was applied for this surface instead, little change in the interface structure was observed within 10 min (Fig. S6 in Appendices). Figure 4(e) shows schematics of the structural change of IL layer. Removal of the IL layer was much slower than its formation as was already discussed for the dissolution experiment in Fig. S4 in Appendices. History-dependent IL interface structure was repeatedly suggested in electro- 

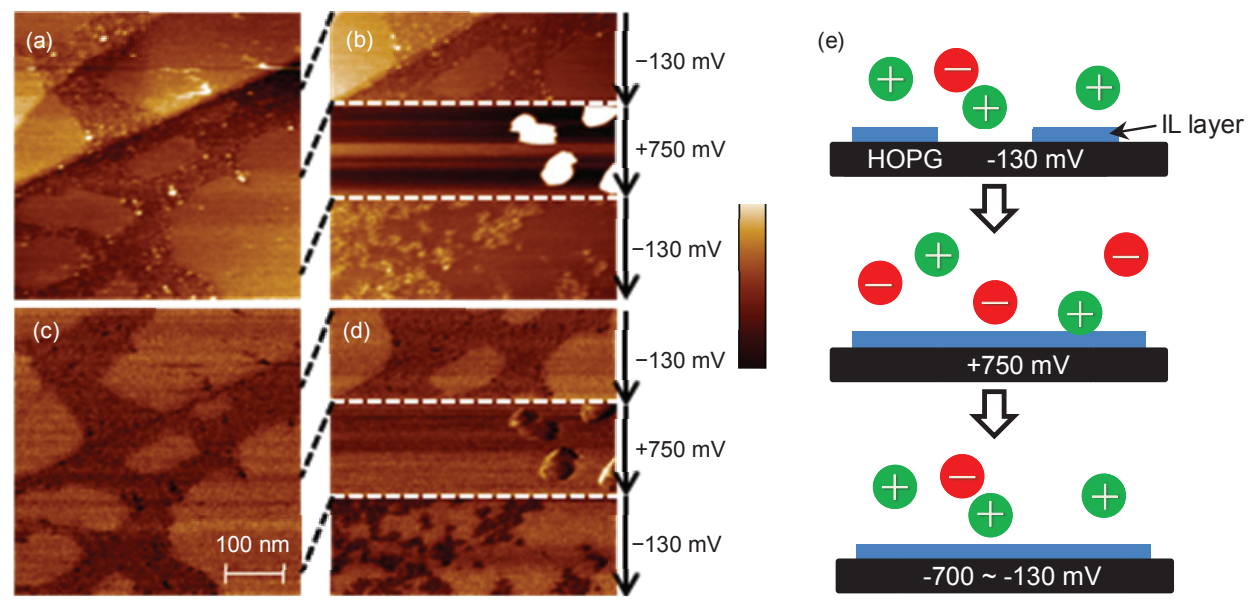

FIG. 4: Topography ((a), (b)) and simultaneously obtained energy dissipation ((c), (d)) EC-FM-AFM images $\left(500 \times 500\right.$ nm ${ }^{2}$, $\Delta f=+175 \mathrm{~Hz}$ ) of an HOPG surface in an IL + water mixture $\left(\chi_{\mathrm{IL}}=0.05\right)$. The electrochemical potential (vs. Pt quasireference electrode) of the substrate $\left(E_{\mathrm{s}}\right)$ was set at $-130 \mathrm{mV}$ (rest potential) for (a) and (c), and changed as $-130 \mathrm{mV}(0-33 \%$ of the scans) $\rightarrow+750 \mathrm{mV}$ (33-66\% of the scans) $\rightarrow-130 \mathrm{mV}$ (66-100\% of the scans) for (b) and (d) while that of the tip ( $\left.E_{\mathrm{t}}\right)$ was fixed at $-110 \mathrm{mV}$ (rest potential). The range of the contrast scale bar for each topography is (a) $1.24 \mathrm{~nm}$ and (b) $3.22 \mathrm{~nm}$, respectively. The contrast scale bar for each energy dissipation ( $V_{\mathrm{Dp}-\mathrm{p}}$ ) image corresponds to (c) 0.382 to $0.403 \mathrm{~V}$, (d) 0.388 to $0.414 \mathrm{~V}$, respectively. Black dotted lines indicate corresponding positions of the images (shifted upwards by thermal drift). (e) Schematics of the irreversible structural change of IL layer.

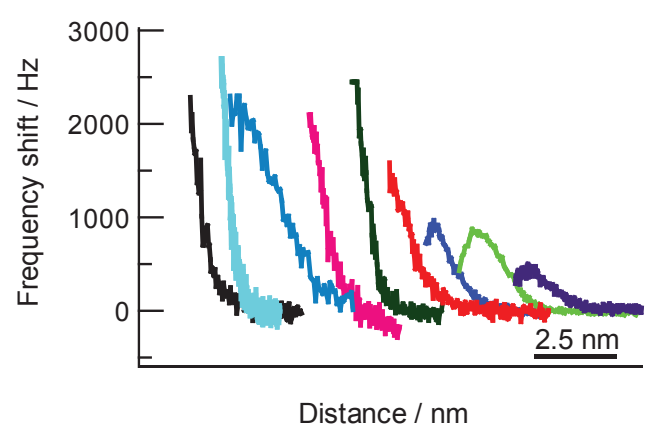

FIG. 5: Frequency shift vs. tip-sample distance curves measured on HOPG in IL + water mixtures at different molar fractions. The curves are laterally shifted to reduce overlapping. The IL molar fraction $\chi_{\mathrm{IL}}$ decreases from right to left as 1 (pure IL), 0.56, 0.35, 0.10, 0.05, 0.02, 0.01, 0.005 and 0 (pure water), respectively.

chemical measurements [45, 46]. Recently, Zhou et al. [47] and Motobayashi et al. [48] have reported the hysteresis of double layer structures of neat IL / metal electrode interfaces upon potential cycling by using vibrational spectroscopy, and revealed that the structure formed at the positive potential requires the large negative potential to recover the original structure. Such a large negative potential is outside of the potential window in the case of IL + water mixtures.

To reveal liquid properties near IL + water / HOPG interfaces, we performed force curve measurements with varying molar fractions. The force curve shows the force acting between the tip and the surface as a function of their separation during approach or retraction. In the case of FM-AFM measurements, we denote the change of frequency shift $(\Delta f)$ vs. distance as a force curve. Force curves shown in Fig. 5 were obtained during approaching the tip to the HOPG surface in IL + water mixtures at various concentrations. The curves were shifted laterally in the order of IL concentration from pure IL at the right end to pure water at the left end to avoid overlap. Lateral axis for each curve is the relative value in each measurement. Actual separation between the tip and the HOPG surface (which onset should be defined as $z=0$ ) could not be determined particularly for $\chi_{\mathrm{IL}}>0.1$ because the oscillation of the cantilever was stopped before it reached to the HOPG surface as will be discussed below. Qualitatively, the positive and negative frequency shifts represent the repulsive and the attractive force, respectively, and the absolute value depends on the resonant frequency, oscillation amplitude, and force constant [49].

A noticeable feature of the force curve for $\chi_{\mathrm{IL}} \geq 0.1$ is the presence of a peak, which is quite different from the force curve with monotonously increased slope up to 2500$3000 \mathrm{~Hz}$ usually observed in aqueous solutions or organic solvents (like the left end curve $\left(\chi_{\mathrm{IL}}=0\right)$ in Fig. 5). After passing the peak, the frequency shift monotonously decreased and usually reached to negative value in a few $\mathrm{nm}$ before the vibration of the cantilever was stopped (even at maximum voltage of our system to drive the piezo actuator for the vibration). Thus, the peak is not a part of structural force due to solvation layers often observed at solid-liquid interfaces $[27,50-52]$. A possible origin of the peak is the higher viscosity of IL at the interface. Ueno et $a l$. have recently reported that ILs confined between two silica surfaces resulted in 1000-fold effective viscosity by resonance shear measurements [19]. By simply applying the equations S3, S5 and S6 in Appendices, increase of viscosity by only $1 \mathrm{mPa}$ s $\left(1 \%\right.$ for the case of $\left.\chi_{\mathrm{IL}}=1\right)$ results in decrease of the resonant frequency by $200 \mathrm{~Hz}$. Thus, even the force acting on the tip apex is repulsive, which should shift the resonant frequency to the positive direc- 

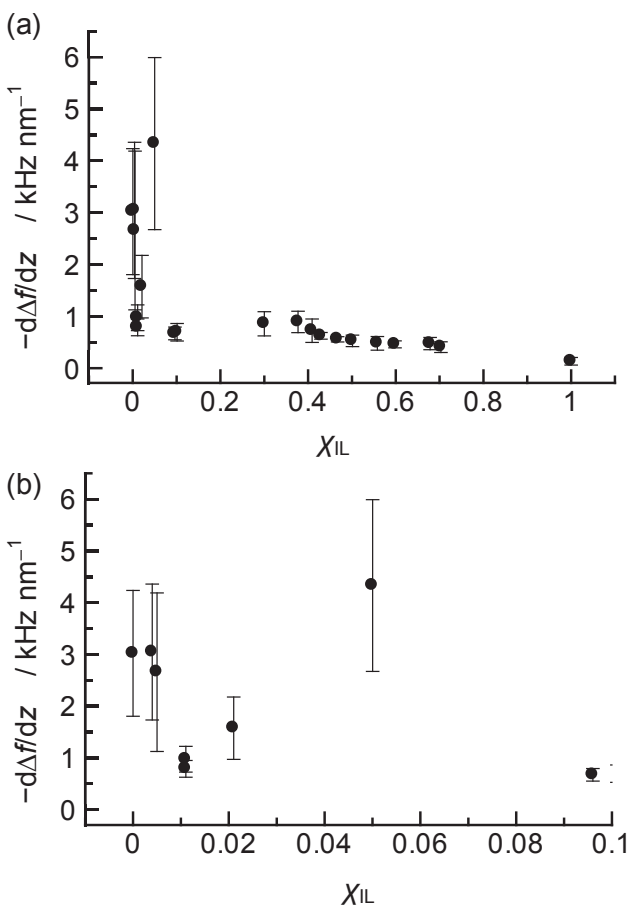

FIG. 6: (a) Gradient of the frequency shift vs. tip-sample distance curves measured on HOPG in IL + water mixtures at different molar fractions. (b) Enlargement of low $\chi_{\mathrm{IL}}$ region of (a). A gradient of a force curve was determined at the region where the curve was assumed to be linear $\left(R^{2}>0.95\right)$. More than ten force curves were measured at different area of the sample to obtain the average gradient in each solution.

tion, negative frequency shift due to higher viscosity at the interface can be dominant. The frequency shift value of the peak was smaller for more viscous (higher $\chi_{\mathrm{IL}}$ ) liquid, suggesting that the viscosity at the interface becomes higher for solution of higher $\chi_{\mathrm{IL}}$. It must be noted that we could not keep the small amplitude vibration $A_{\mathrm{p}-\mathrm{p}}$ while the tip invaded into the IL layer. It is a striking difference from force curve measurements in contact-mode AFM, where equidistant drops of force corresponding to the breaking of an IL layer by the tip were observed [815]. Therefore, FM-AFM could image very outer layer in topography and trace only outer region by a force curve.

The gradient of each force curve $(-d \Delta f / d z)$ at its linear region was calculated to compare the steepness in different IL + water mixtures (Fig. 6(a)). For the solutions of $\chi_{\mathrm{IL}} \geq 0.1$, the gradient gradually decreased for higher $\chi_{\mathrm{IL}}$. Higher viscosity at the interface and its dependency on $\chi_{\mathrm{IL}}$ of bulk liquid relates to the change as already discussed (Fig. 7(b)). Scattered gradient data were found at $\chi_{\mathrm{IL}}<0.1$ (Fig. 6(b)). None of the force curves for $\chi_{\mathrm{IL}}<0.1$ had a peak, thus the increase of viscosity was probably not a dominant factor for the gradient value of the linearly increased region for these diluted solutions. The gradient value and its error bar for the solution of $\chi_{\mathrm{IL}}=0.005$ were similar to those of pure water $\left(\chi_{\mathrm{IL}}=0\right)$ or an $\mathrm{HClO}_{4}$ aqueous solution (not shown). Imaging of the interface was quite difficult for the range of $0.005<$ $\chi_{\mathrm{IL}} \leq 0.01$ as noted above, but the gradient of the force curve for $\chi_{\mathrm{IL}}=0.01$ showed a small error bar at a rather

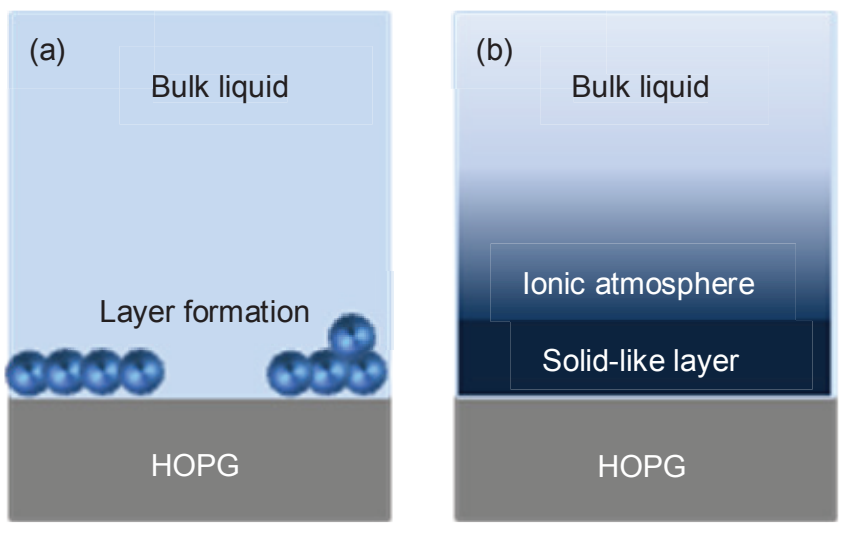

FIG. 7: Schematic illustration of IL + water / HOPG interfaces. (a) $0.01<\chi_{\mathrm{IL}} \leq \mathrm{CC}$, (b) $\mathrm{CC}<\chi_{\mathrm{IL}} \leq 1$. CC is 0.05-0.1.

small value of about $1 \mathrm{kHz} \mathrm{nm}^{-1}$. It may be due to high concentration of IL agglomerates on the HOPG surface. The unstructured agglomerates at the interface can show a blunt response against the approaching tip.

The solution of $\chi_{\mathrm{IL}}=0.05$ was close to the threshold concentration (TC) for formation of an IL layer at the interface, where both of IL covered region and bare HOPG region could be identified by imaging (Figs. 2 (c) and (h), and Fig. S3 in Appendices). A critical concentration (CC), where the HOPG surface is covered by a single IL layer exists between $\chi_{\mathrm{IL}}=0.05$ and 0.1 . For the solution concentration below CC, the HOPG surface is partially covered by a single IL layer (Fig. 7(a)) or unstructured IL agglomerates (particularly at $\chi_{\mathrm{IL}}<\mathrm{TC}$ ). The inhomogeneity probably a factor for rather a large error bar for $\chi_{\mathrm{IL}}=0.05$.

The bulk properties of IL + water mixtures have been investigated using various methods such as calorimetry, vapor pressure measurements, and IR absorption [33, 38, 39]. Koga et al. suggested that the constituent ions were completely dissociated only in low concentration IL + water mixtures of BMIM-BF 4 and BMIM-I ('critical aggregation concentration' (CAC) was $\chi_{\mathrm{IL}}=0.013-0.015$ ), and association of counterions above the threshold led to formation of IL clusters in the range $\chi_{\mathrm{IL}}>0.6$ [33]. Jeon et al. proposed that BMIM-BF ${ }_{4}$ started to form micelles or aggregates at $c_{\text {water }}=32$ and $45 \mathrm{~mol} \mathrm{l}^{-1}\left(\chi_{\mathrm{IL}} \approx 0.02\right.$ and 0.06) [39]. Our proposed concentration of the onset of IL layer formation (TC, $\chi_{\mathrm{IL}} \sim 0.05$ ) is close to the value proposed by Jeon et al., which may indicate that the onset of counterion interaction in the bulk of BMIM$\mathrm{BF}_{4}$ aqueous solution leads to the IL layer formation on HOPG. An IL + water mixture (BMIM-BF 4 aqueous solution) / air interface was investigated by surface tension and sum-frequency generation (SFG) spectroscopy measurements by Ouchi et al. [35, 53]. They found that the surface of a highly diluted IL + water mixture was mostly covered with BMIM cations, and a threshold concentration for $\mathrm{BF}_{4}$ anions to appear at the surface was in the range of $0.016 \leq \chi_{\mathrm{IL}} \leq 0.05$ [35], which is also close to the value we obtained in the present work. 


\section{CONCLUSIONS}

We have performed FM-AFM measurements of HOPG

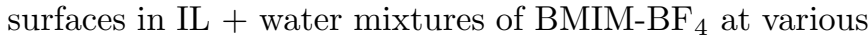
molar fractions. FM-AFM measurements showed that stable IL layers completely covered the HOPG surface even for diluted IL + water mixtures as low as $\chi_{\mathrm{IL}}=0.1$. The $0.68 \mathrm{~nm}$-high steps, which corresponded to the ion pair size of BMIM-BF 4 , were observed as in the case of pure IL $\left(\chi_{\mathrm{IL}}=1.0\right)$. Only at $\chi_{\mathrm{IL}}=0.05$, coexistence of IL covered region and bare HOPG region (partially covered by a single IL layer) was confirmed by different contrast in the energy dissipation image across the step of $0.68 \mathrm{~nm}$ high. The critical concentration for the completion of a single IL layer on HOPG was between $\chi_{\mathrm{IL}}=0.05$ and 0.1 . Force curve measurements in a series of IL + water mixtures also suggested that the liquid side nature such as the apparent viscosity near the interfaces changed around the critical concentration. Removal of the IL layer from the HOPG surface was found to be a slow process, suggesting that the interface structure does not necessarily show a quick response against a change in the liquid phase concentration. Furthermore, electrochemical potential of the HOPG substrate affected the IL layer formation. Particularly application of positive potential resulted in growth of IL layer at the interface rather than a preferential adsorption of anions by application of the positive potential.

\section{Acknowledgments}

We thank Prof. Toshiaki Enoki and Dr. Kazuyuki Takai for a gift of HOPG substrates. This study was supported by the Funding Program for Next Generation World-Leading Researchers (NEXT Program, GR071) of the Japan Society for the Promotion of Science (JSPS) and Grant-in-Aid (No. 20001006) from the Ministry of Education, Culture, Sports, Science, and Technology, Japan.

\section{Appendices}

The details of resonant frequency in solutions, a method of molar ratio calibration, the histograms of step height, FM-AFM images of the HOPG surface in IL + water mixture $\left(\chi_{\mathrm{IL}}=0.05\right)$ and in air after removal of dropped IL + water mixture liquid phase and after following drop and removal of water droplets, cyclic voltammograms, and an additional EC-FMAFM images are available in Supplementary materials (https://www.jstage.jst.go.jp/article/ejssnt/12/0/12_89/ _article/supplement).
[1] M. Armand, F. Endres, D. R. MacFarlane, H. Ohno, and B. Scrosati, Nat. Mater. 8, 621 (2009).

[2] H. Liu, Y. Liu, and J. Li, Phys. Chem. Chem. Phys. 12 1685 (2010).

[3] T. Torimoto, T. Tsuda, K. Okazaki, and S. Kuwabata, Adv. Mater. 22, 1196 (2010).

[4] T. Uemura, R. Hirahara, Y. Tominari, S. Ono, S. Seki, and J. Takeya, Appl. Phys. Lett. 93, 263305 (2008).

[5] K. R. J. Lovelock, I. J. Villar-Garcia, F. Maier, H.-P. Steinruck, and P. Licence, Chem. Rev. 110, 5158 (2010).

[6] J. B. Rollins, B. D. Fitchett, and J. C. Conboy, J. Phys. Chem. B 111, 4990 (2007).

[7] M. Mezger, H. Schroder, H. Reichert, S. Schramm, J. S. Okasinski, S. Schoder, V. Honkimaki, M. Deutsch, B. M. Ocko, J. Ralston, M. Rohwerder, M. Stratmann, and H. Dosch, Science 322, 424 (2008).

[8] R. Atkin and G. G. Warr, J. Phys. Chem. C 111, 5162 (2007).

[9] R. Atkin, N. Borisenko, M. Druschler, S. Z. E. Abedin, F. Endres, R. Hayes, B. Huber, and B. Rolling, Phys. Chem. Chem. Phys. 13, 6849 (2011).

[10] D. Wakeham, R. Hayes, G. G. Warr, and R. Atkin, J. Phys. Chem. B 113, 5961 (2009).

[11] R. Hayes, S. Z. E. Abedin, and R. Atkin, J. Phys. Chem. B 113, 7049 (2009).

[12] R. Atkin, S. Z. E. Abedin, R. Hayes, L. H. S. Gasparotto, N. Borisenko, and F. Endres, J. Phys. Chem. C 113, 13266 (2009).

[13] R. Hayes, G. G. Warr, and R. Atkin, Phys. Chem. Chem. Phys. 12, 1709 (2010).

[14] R. Hayes, N. Borisenko, M. K. Tam, P. C. Howlett, F. Endres, and R. Atkin, J. Phys. Chem. C 115, 6855 (2011).

[15] J. J. Segura, A. Elbourne, E. J. Wanless, G. G. Warr, K. Voitchovsky, and R. Atkin, Phys. Chem. Chem. Phys. 15, 3320 (2013).
[16] Y. Liu, Y. Zhang, G. Wu, and J. Hu, J. Am. Chem. Soc. 128, 7456 (2006).

[17] S. Bovio, A. Podesta, C. Lenardi, and P. Milani, J. Phys. Chem. B 113, 6600 (2009).

[18] Z. Fuchun, S. Maolin, R. Xiuping, W.Guozhong, H. Jun, and Z. Yi, Chin. Phys. Lett. 27, 086101 (2010).

[19] K. Ueno, M. Kasuya, M. Watanabe, M. Mizukami, and K. Kurihara, Phys. Chem. Chem. Phys. 12, 4066 (2010).

[20] S. Maolin, Z. Fuchun, W. Guozhong, F. Haiping, W. Chunlei, C. Shimou, Z. Yi, and H. Jun, J. Chem. Phys. 128, 134504 (2008).

[21] S. A. Kislenko, I. S. Samoylov, and R. H. Amirov, Phys. Chem. Chem. Phys. 11, 5584 (2009).

[22] S. A. Kislenko, R. H. Amirov, and I. S. Samoylov, Phys. Chem. Chem. Phys. 12, 11245 (2010).

[23] S. Wang, S. Li, Z. Cao, and T. Yan, J. Phys. Chem. C 114, 990 (2010).

[24] C. Schroder, T. Rudas, and O. Steinhauser, J. Chem. Phys. 125, 244506 (2006)

[25] J. Vatamanu, O. Borodin, and G. D. Smith, J. Am. Chem. Soc. 132, 14825 (2010).

[26] Y. Yokota, T. Harada, and K. Fukui, Chem. Commun. 46, 8627 (2010).

[27] T. Fukuma, K. Kobayashi, K. Matushige, and H. Yamada, Appl. Phys. Lett. 87, 034101 (2005).

[28] K. Umeda and K. Fukui, Langmuir 26, 9104 (2010).

[29] T. Fukuma, Y. Ueda, S. Yoshioka, and H. Asakawa, Phys. Rev. Lett. 104, 016101 (2010).

[30] T. Hiasa, K. Kimura, and H. Onishi, J. Phys. Chem. C 116, 26475 (2012).

[31] T. Ichii, M. Fujimura, M. Negami, K. Murase, and H. Sugimura, Jpn. J. Appl. Phys. 51, 08KB08 (2012).

[32] Y. Yokota, H. Hara, T. Harada, A. Imanishi, T. Uemura, J. Takeya, and K. Fukui, Chem. Commun. 49, 10596 (2013). 
[33] H. Katayanagi, K. Nishikawa, H. Shimozaki, K. Miki, P. Westh, and Y. Koga, J. Phys. Chem. B 108, 19451 (2004).

[34] I. B. Malham, P. Letellier, and M. Turmine, J. Phys. Chem. B 110, 14212 (2006).

[35] J. Sung, Y. Jeon, D. Kim, T. Iwahashi, K. Seki, T. Iimori, and Y. Ouchi, Colloids and Surf. A 284, 84 (2006).

[36] S. Saha and H. Hamaguchi, J. Phys. Chem. B 110, 2777 (2006).

[37] W. Jiang, Y. Wang, and G. A. Voth, J. Phys. Chem. B 111, 4812 (2007).

[38] H. Kato, K. Nishikawa, H. Murai, T. Morita, and Y. Koga, J. Phys. Chem. B 112, 13344 (2008).

[39] Y. Jeon, J. Sung, D. Kim, C. Seo, H. Cheong, Y. Ouchi, R. Ozawa, and H. Hamaguchi, J. Phys. Chem. B 112, 923 (2008).

[40] R. G. Horn, D. F. Evans, and B. W. Ninham, J. Phys. Chem. 92, 3531 (1988).

[41] J. A. Smith, O. Werzer, G. B. Webber, G. G. Warr, and R. Atkin, J. Phys. Chem. Lett. 1, 64 (2010).

[42] E. Rilo, J. Vila, J. Pico, S. Garcia-Garabal, L. Segade, L. M. Varela and O. Cabeza, J. Chem. Eng. Data 55, 639 (2010).

[43] M. Fujihira, Y. Okabe, Y. Tani, M. Furugori, and U. Akiba, Ultramicroscopy 82, 181 (2000).
[44] S. Morita, R. Wiesendanger, and E. Meyer (Eds.), Noncontact Atomic Force Microscopy (Springer-Verlag, Berlin, 2002).

[45] M. Druschler, N. Borisenko, J. Wallauer, C. Winter, B. Huber, F. Endres, and B. Roling, Phys. Chem. Chem. Phys. 14, 5090 (2012).

[46] N. Nishi, Y. Hirano, T. Motokawa, and T. Kakiuchi, Phys. Chem. Chem. Phys. 15, 11615 (2013).

[47] W. Zhou, S. Inoue, T. Iwahashi, K. Kanai, K. Seki, T. Miyamae, D. Kim, Y. Katayama, and Y. Ouchi, Electrochem. Commun. 12, 672 (2010).

[48] K. Motobayashi, K. Minami, N. Nishi, T. Sakka, and M. Osawa, J. Phys. Chem. Lett. 4, 3110 (2013).

[49] N. Sasaki and M. Tsukada, Jpn. J. Appl. Phys. 39, L1334 (2000).

[50] H. K. Christenson, J. Phys. Chem. 90, 4 (1986).

[51] W. Hofbauer, R. J. Ho, R. Hairulnizam, N. N. Gosvami, and S. J. O'shea, Phys. Rev. B 80, 134104 (2009).

[52] T. Hiasa, K. Kimura, H. Onishi, M. Ohta, K. Watanabe, R. Kokawa, N. Oyabu, K. Kobayashi, and H. Yamada, J. Phys. Chem. C 114, 21423 (2010).

[53] J. Sung, Y. Jeon, D. Kim, T. Iwahashi, T. Iimori, K. Seki, and Y. Ouchi, Chem. Phys. Lett. 406, 495 (2005). 